\title{
LA ESTRATEGIA DEL PP Y PSOE PARA INCORPORAR A LAS MUJERES EN LA POLÍTICA NACIONAL (1979-2008)
}

\section{THE STRATEGY OF PP AND PSOE TO INCORPORATE WOMEN IN NATIONAL POLITICS (1979-2008)}

\author{
Gema Sánchez Medero \\ Universidad Complutense, Madrid. España/Spain \\ gsmedero@.cps.ucm.es
}

Recibido/Received: 22/05/2010

Aceptado/Accepted: 25/07/2010

\section{RESUMEN}

En el presente artículo hemos analizado las políticas que han desarrollado los dos grandes partidos de ámbito nacional en España, PP y PSOE, para incorporar a las mujeres a la vida política, y alcanzar una participación equilibrada entre hombres y mujeres. De esta forma, podremos averiguar el verdadero impacto que tienen las mismas y el papel que hasta este momento han desempeñado en los partidos objeto de estudio, con el único fin de conocer si este tipo de estrategias han supuesto una mera campaña marketing, o si por el contrario han ido más allá. Se trata de dictaminar si realmente las medidas de discriminación positiva son efectivas o no.

\section{PALABRAS CLAVE}

Democracia interna, afiliados, PP, PSOE, poder de decisión y participación.

\section{SUMARIO}

1. Introducción. 2. Las mujeres y la política. 3. La estrategia paritaria de los partidos políticos españoles. 4. Evaluación del grado de participación femenina en los órganos nacionales del partido. 5. Evaluación del grado de participación femenina en los cargos de representación nacional. 6. Conclusiones. Bibliografía.

\begin{abstract}
In this paper we have analyzed the policies they have developed two major national parties in Spain, PP and PSOE, to bring women into political life, and achieve a balance between men and women. In this way, we can ascertain the real impact of the same and the role they have played so far in the matches under study, with the sole purpose of knowing this type of strategies have been a simple marketing campaign, or whether otherwise have gone further. This is really decided whether affirmative action is effective or not.
\end{abstract}

\section{KEYWORDS}

Internal democracy, affiliates, PP, PSOE, decision powers and participation.

\section{CONTENTS}

1.Introduction. 2. Women and politics. 3. The joint strategy of the Spanish political parties. 4. Evaluation of the degree of female participation in the national party organs. 5. Evaluation of the degree of female participation in positions of national representation. 6. Conclusions. References. 


\section{INTRODUCCIÓN}

Los estudios sobre la mujer en la vida política casi siempre han estado centrados en el análisis de la participación de éstas en las instituciones políticas, principalmente el Parlamento, descuidando con ello otro aspecto tal vez tan importante dentro del poder político como es la presencia de las mujeres en los órganos ejecutivos de los partidos políticos. Por eso nosotros nos hemos decantado por analizar desde una perspectiva global las medidas que han adoptado PP y PSOE para fomentar la participación de las mujeres en la vida política española. De ahí, que nos hayamos centrado en averiguar el verdadero alcance que han tenido dichas acciones a la hora de integrar a las mujeres en sus órganos de gobierno $\mathrm{y}$ en las diferentes arenas políticas. Al mismo tiempo, hemos intentado estudiar el impacto de la Ley de Igualdad aprobada por el gobierno socialista. El objetivo era conocer si su presencia en el ámbito político respondía más a un carácter simbólico que real, es decir, si las mujeres forman parte y participan de los núcleos de poder donde se toman verdaderamente las decisiones que rigen la vida de la organización. Así, para tal fin hemos optado por el estudio de modelos diferentes. Uno, el del PSOE, que ha sido la primera fuerza política que en España se decantó por un sistema de cuotas y, otro, el del PP, por ser el único partido con implantación nacional que no ha dispuesto ninguna medida estatutaria que venga a favorecer la inclusión de las mujeres. Para ello, nos hemos fundamentado un doble tratamiento metodológico, por un lado, la identificación de las tareas que desempeñan en su actividad política y las estrategias de los distintos partidos y, por otro, los cálculos descriptivos sobre la proporción de mujeres que ocupan posiciones políticas en los distintos niveles de poder.

\section{LAS MUJERES Y LA POLÍTICA}

Desde la Antigüedad, la política ha sido un área de la vida pública que ha estado reservada para los hombres hasta que a principios del siglo XX se ampliara el sufragio. Aunque, pese a ello, la participación de las mujeres en política ha sido más bien escasa si tenemos en cuenta el volumen de población que representan. Habría que preguntarse si éstas muestran realmente un desinterés por integrarse en la arena política, o si por el contrario, es la propia sociedad quién al efectuar el reparto de roles y responsabilidades les ha relegado a otro tipo de actividades. Aunque también es cierto que esta situación se ha ido modificando paulatinamente por la propia demanda de la población femenina, que ha exigido a las elites políticas una serie de reformas que en algunos países han supuesto incluso la aparición de nuevos temas en los programas de los partidos, nuevos sistemas para la selección de candidaturas, nuevas formas de elaboración de política públicas y el establecimiento de nuevas estructuras de gobierno como ministerios para las mujeres. Se trata en definitiva de articular fórmulas que fomenten la inclusión de la mujer en la vida política. Con tal motivo, los partidos se han visto ante la obligación de desarrollar tres tipos de estrategias: la primera, aceptar las demandas de las mujeres e incorporarlas a su discurso, además de reconocer públicamente la importancia de aumentar su presencia; la segunda, animarlas a participar más activamente en política; y la tercera, potenciar las medidas que favorecen la discriminación positiva.

Todo, porque las mujeres constituyen cerca del 50\% del electorado, por lo que desoír las peticiones de este grupo de votantes podría tener consecuencias negativas para 
cualquier partido que posea aspiraciones de gobierno (Ruiz Jiménez, 2002). Tampoco hay que obviar las transformaciones que se están produciendo en la propia organización social, que conduce a la sustitución del modelo tradicional dominante (desenvolvimiento en la esfera de la familia, de lo privado) por un nuevo modelo femenino (mujer que trabaja, desenvolvimiento en la esfera pública) (García de León, 1991:11). Ambos hechos han contribuido a que la mujer se incorpore poco a poco a la vida política, hasta el punto que en 1990 sólo había ocho mujeres que estuvieran al frente de ejecutivos nacionales y diez años más tarde, este número se ha visto sustancialmente incrementado, y lo que es más importante empiezan a conseguir mayor presencia en cargos de máxima responsabilidad en países de cultura, tradiciones, historia y religión (Cernuda, 2000:8).

Hay que mencionar también que la participación femenina en la vida política ha aumentado gracias a una serie de medidas que se adoptan tanto desde las instituciones públicas como desde las organizaciones políticas. Éstas consisten principalmente en el establecimiento de cuotas, en la búsqueda de mecanismos que les permitan formar parte activa de la vida política como son las medidas dirigidas a la conciliación de la vida familiar y política, o la regulación por ley de la paridad. Son propuestas que van encaminadas a garantizar la participación equilibrada de varones y mujeres en los órganos políticos, es lo que las feministas francesas ha denominado parité (paridad), que significa igualdad numérica. Aunque la tendencia actual, marcada en la I Cumbre Europea de Mujeres es apostar por la "democracia paritaria". En todo caso, y pese a esto, es necesario establecer un sistema de cuotas que asegure y potencie la participación femenina. Tradicionalmente han sido los partidos de izquierda quiénes han llevado a la práctica el sistema de cuotas, mientras que los partidos de derecha han utilizado estrategias más retóricas para animar la participación femenina (Uriarte y Elizondo, 1997: 107). Así, por ejemplo, en España el Partido Socialista Obrero Español (PSOE) ha sido el primer partido que ha promovido estatutariamente la inclusión de mujeres en sus órganos de dirección y aprobado en la actual legislatura, incluso, una Ley de Igualdad de Género.

\section{LA ESTRATEGIA PARITARIA DE LOS PARTIDOS POLÍTICOS ESPAÑOLES}

Todos los partidos políticos españoles desarrollan, en mayor o menor medida, algún tipo de estrategia para incorporar a mujeres en sus organizaciones, entre las mismas cabe destacar las denominadas "discursivas" o "retóricas", las de "acción positiva" y las de "discriminación positiva". Todo, porque los partidos políticos, se ven en la obligación de introducir temas de género y hacerse eco de las demandas de un sector que representa más o menos la mitad de la población mundial, ya que si los partidos políticos quieren competir electoralmente no pueden ser ajenos a las peticiones de este grupo de votantes, y más si aspiran a acceder al Gobierno de la nación. De esta forma, no cabe duda que los objetivos intervienen de forma drástica en el proceso de toma de decisiones de los partidos políticos (Hall, 1973: 308), además de convertirse en el principal referente del cambio de estrategia. Con lo cual, estamos admitiendo que, en muchos casos, el estímulo al cambio es eminentemente externo, y posiblemente el más frecuente sea el fracaso electoral (Katz y Mair, 1994: 18). Por tanto, cuanto más pronunciada sea la derrota electoral en los partidos cuyo objetivo principal sea ganar las elecciones, mayor será la probabilidad de que éstos emprendan cambios (Harmel y 
Janda, 1994), es decir, cuanto mayor sea el revés externo que sufra un partido, mayor será la propensión a una reestructuración que ponga en marcha el partido (Panebianco, 1990).

Si nos atenemos a esto, cabría pensar que los cambios únicamente responden a la derrota electoral, o a una previsión de ella, dado que los miembros de dichas organizaciones se muestran reacios a cualquier modificación, sino existe una poderosa razón para ello. Aunque en el caso que aquí estamos tratando no responde solamente a esta lógica, ya que en AP/PP los cambios más relevantes se han emprendido tras una mejora en los resultados electorales, $\mathrm{y}$ en el PSOE se han producido cuando se encontraban gobernando a nivel nacional tras dos mayorías absolutas consecutivas. De ahí, que los cambios efectuados no coincidan totalmente con una modificación del objetivo principal ni con una derrota electoral, sino que también han obedecido al propio interés que tienen ambos partidos por atraer para sí al electorado femenino. Y de ahí, que se hayan decidido a: introducir en su discurso temas de interés para la población femenina, incrementar la presencia de mujeres en los órganos de dirección, o articular medidas que favorezcan la paridad, por poner algunos ejemplos.

La primera, de ellas, hace referencia a como las fuerzas políticas incorporan en su discurso y programa contenidos que tienden a favorecer la equidad entre hombres y mujeres. En el PSOE, por ejemplo, la agenda de la igualdad estaba dominada por las medidas que guardaban relación con el mercado laboral y la educación o las políticas sociales, pero con el tiempo el tema de la representación ha pasado a ser prioritario. De ahí, que los socialistas se hayan dedicado a realizar propuestas para fomentar el asociacionismo femenino y para conseguir una participación paritaria en los órganos representativos y de toma de decisiones en los ámbitos políticos, sociales y culturales, incluyendo la reforma del procedimiento administrativo de contratación funcionarial, la reforma de la Ley Electoral para garantizar una presencia equilibrada en las listas, la configuración de gobiernos paritarios o la eliminación de la discriminación sobre la mujer en el acceso a la Jefatura del Estado (Verge, 2005). El PP, en cambio, no ha tomado partido a este respecto hasta 1993, cuando se manifiesta a favor, por primera vez, de la ampliación de la participación de las mujeres en la vida económica y social (PP, 1993:21). No obstante, después, en las elecciones de 1996, se declaró como objetivo irrenunciable la plena igualdad de la mujer en la sociedad española. Aunque este principio de intenciones no tiene reflejo en los posteriores programas electorales, y sólo parece primar en una parte del discurso popular.

La segunda estrategia tiene relación con los cambios que se producen en la estructura o en las actividades formativas de los partidos políticos en correlación con el tema de igualdad de género (Verge, 2006:169). En el PSOE, la preocupación por incrementar la militancia femenina ha sido una constante en todo el período estudiado, aunque esta voluntad no se haya materializado siempre en campañas concretas (Verge, 2006:169). Los socialistas aprobaron en su XXXI Congreso Federal (1988) el lanzamiento de una campaña de afiliación femenina "Cada una, una" y la famosa cuota de representación del $25 \%$ de mujeres en sus órganos ejecutivos. Un año más tarde, la Secretaría General de la Mujer, articuló lo que vino a denominarse "Zonas Urgentes de Afiliación", con el fin de fomentar la inclusión de las mujeres en la política. Estas medidas han contribuido a que la tasa de afiliación femenina haya pasado de un 24,64\% en 1996 a un 28,40\% en 2001 y en el 2006 ha llegado a superar el $30 \%$ (Ver tabla 1). No obstante, y a pesar de los esfuerzos que están realizando por integrar a las mujeres dentro de la organización, las cifras se encuentran todavía muy lejos de lo que sería una militancia equilibrada entre sexos. La situación en el PP, como muestra la Tabla 1, no es mucho mejor: en 1996 las mujeres populares representaban el 29,7\% sobre la afiliación 
del partido, aventajando a los socialistas en 5 puntos. Sin embargo, el crecimiento experimentado por el PP es menor que en el PSOE, ya que entre 1996 y 2001 la afiliación ha crecido en 4,98 puntos, mientras que los socialistas lo han hecho en 7,86.

Pese a ello, también es necesario advertir otro dato curioso, el PP disfruta de mayores porcentajes de afiliación femenina, aunque sea sólo por una pequeña diferencia. La razón es que normalmente las mujeres de esta fuerza política suelen proceder de clases acomodadas, lo que les permite liberarse con mayor facilidad del trabajo doméstico (Verge, 2006: 170). También porque los partidos de izquierda se han desarrollado en estrecha colaboración con los sindicatos y el mundo del trabajo, ámbitos a los que la mujer se ha incorporado mucho más tarde que el hombre (Verge, 2006: 171). En todo caso, como decimos, es cierto que los partidos de derecha presentan unos mayores porcentajes de afiliación femenina, aunque, en los últimos años, parece que se están equiparando.

Tabla 1. La afiliación femenina en el PSOE y PP

\begin{tabular}{|l|c|c|c|c|c|c|c|c|c|c|}
\cline { 2 - 11 } \multicolumn{1}{c|}{} & \multicolumn{2}{c|}{$\mathbf{1 9 9 6}$} & \multicolumn{2}{c|}{$\mathbf{2 0 0 1}$} & \multicolumn{2}{c|}{$\mathbf{2 0 0 2}$} & \multicolumn{2}{c|}{$\mathbf{2 0 0 5}$} & $\mathbf{2 0 0 6}$ & $\mathbf{2 0 0 7}$ \\
\cline { 2 - 5 } & Mujes & $\%$ & Mujeres & $\%$ & Mujeres & $\%$ & Mujeres & $\%$ & $\%$ & $\%$ \\
\hline PSOE & 90.227 & 24,64 & 119.003 & 28,40 & 145.296 & 30 & 486.334 & 31,72 & 32,5 & --- \\
\hline PP & 149.798 & 29,70 & 207.231 & 32,76 & ----- & 32,8 & ---- & ---- & --- & 34,68 \\
\hline
\end{tabular}

Fuente: Elaboración propia a partir de los datos facilitados por el Instituto de la Mujer

Además, hay que señalar, que los socialistas conceden mucha importancia a la formación política de las afiliadas, hasta el punto que obligan a todos aquellos que ingresan en la organización a realizar un curso de formación previa, aunque también existen, de forma sistemática, programas de ciclos formativos. Lo que se pretende con ello es evitar que el mayor reclutamiento de mujeres sea cuestionado por cierto sectores del partido debido, en principio, a la menor educación política de las militantes como elevar la autoestima de las propias afiliadas (Verge, 2006: 171). En el PP, en cambio, no conocemos de la existencia de ciclos formativos específicos para las afiliadas, a parte, de las actividades que sean programadas por la Asociación Democrática Conservadora.

Por otra parte, los partidos políticos también se han visto obligados a crear secciones, áreas y organizaciones femeninas para responder y hacerse eco de las demandas de este importante sector de la población. Aunque habría que advertir que en el Partido Socialista no existe una organización femenina al uso, pero sí cuenta con una Secretaría de la Mujer que se encarga de presentar iniciativas a los órganos directivos del partido (Cernuda, 2000: 66). Eso sí, anteriormente a la constitución de esta secretaría si se podía hablar de una especie de organización femenina, los grupos que originalmente se denominaron "Mujer y Opción Socialista", y más tarde "Mujer y Socialismo", que perduraron hasta que se conformo la Secretaría de Participación de la Mujer en 1985. Ésta se encontraba integrada hasta 1990 en la Secretaría de Movimientos Sociales y Participación Ciudadana, año en que se constituirá finalmente en Secretaría de Área Federal, consiguiendo así un rango mucho mayor en la estructura orgánica del partido (desde 2000, Secretaría de Igualdad, y actualmente, Secretaría de Área de Política e Igualdad). Para la consecución de sus objetivos, la mencionada Secretaría buscó la profundización de las relaciones con el movimiento feminista, el desarrollo de la organización de las mujeres y la promoción de la participación de las militantes socialistas en estas asociaciones, para hacer frente tanto a la tradicional hegemonía 
conservadora en este sector como a movimientos feministas más radicales y opuestos a la participación institucional que no mostraban ningún apoyo a las políticas para la mujer diseñadas desde el Gobierno del PSOE (Verge, 2006: 172). Este respaldo a la organización de las mujeres se plasmó en 1987 en la creación de la Federación de Mujeres Progresistas (FMP) y en la ayuda que les prestó en todo momento la Internacional Socialista de Mujeres que ha jugado un papel crucial en la promoción de las cuotas entre los partidos que están integrados en ella. Además, José Luis Rodríguez Zapatero tras su reelección al frente del Gobierno de la Nación decidió crear el Ministerio de la Igualdad e Investigación y Desarrollo, para que también desde el ejecutivo se propongan medidas que vayan destinadas a estos fines, por no hablar, de la ya conocida Ley de Igualdad.

El PP, siempre ha contado con un departamento que se ha encargado de los temas de la mujer. Fue la presidencia de Manuel Fraga la que impulsó la creación de una organización de mujeres, los motivos fueron dos muy concretos: 1) trabajar en las cuestiones relacionadas con los problemas de las mujeres y la familia, y 2) crear una estructura bien organizada que sirviera no sólo como cantera para promocionar mujeres en puestos de responsabilidad sino también como reclamo para lograr captar el voto femenino (Cernuda, 2000:63). De esta forma, nació en 1982 la organización "Mujeres Conservadoras", presidida por María Antonia Suárez, aunque fue Isabel Tocino quién la dotó de una estructura territorial y la cambió de nombre por "Asociación Democrática Conservadora". A partir de entonces funciona como una ONG convencional que recibe subvenciones de distintos organismos, sobre todo el Ministerio de Trabajo y Asuntos Sociales (Sánchez Medero, 2007:106). Pero, además, el PP ha contado también con una secretaría para temas relacionados con la igualdad de género. Así, a partir de 1989, se constituye la Secretaría dentro del Área de Política Social (desde 1999, Secretaría de Igualdad de Oportunidades y Educación). Aunque hasta finales de los años noventa, las relaciones con las organizaciones de mujeres y la elaboración de las políticas de la mujer estaban bajo la responsabilidad del Grupo Popular en el Congreso de los Diputados (Verge, 2006:172). Esto, unido al hecho de que la mencionada secretaría se encuentre ubicada dentro del área social, indica la poca autonomía que tienen los temas del género en este partido.

La tercera estrategia, la de la "discriminación positiva", hace mención a las modificaciones que se han introducido en los estatutos de los partidos para apoyar o impulsar una mayor presencia de las mujeres en los cargos partidistas e institucionales. En este sentido cabría señalar, que de los tres partidos de ámbito nacional, el único que no contempla en su normativa ninguna política de discriminación positiva es el PP, pero eso no significa que los populares no apuesten por la incorporación de mujeres en los cargos directivos. Es más, se podría llegar a sostener que gracias a la renovación emprendida por el partido, el número de éstas, tanto en los órganos directivos como en los cargos de representación institucional, se ha visto incrementado de manera considerable, lo que nos induce a pensar que pese a todo, los populares han practicado un sistema de cuotas encubierto. Por mucho que ellos se empeñen en manifestar que en el PP los cargos femeninos responden a "la nota y no a la cuota" (PP, 2002:148), es decir, en las capacidades personales y en el mérito individual. Porque si eso fuera así realmente, cómo se podría explicar, por ejemplo, que en el XIV Congreso Nacional (2002) la disposición de los ponentes al mismo fuera hombre y mujer, es decir, 50 y 50 . La cuestión es que los populares quieren demostrar, que a diferencia de otros partidos, no recurren a técnicas de marketing político, como ellos consideran que son las "cuotas", para contar con una alta presencia de mujeres en sus órganos de dirección y en sus 
listas electorales. Además, suelen recordar que el Gobierno de José María Aznar fue el primero en designar a mujeres en áreas que antes estaban copadas por hombres, como los ministerios, la presidencia del Congreso de los Diputados y del Senado, o la Vicepresidencia de la Unión Europea. Y pese a no disponer medidas de discriminación positiva el Partido Popular ha pasado de ser uno de los partidos con menor representación femenina a ser uno donde las mujeres cuentan con mayor presencia.

Pero el rechazo de los populares a la política de "cuotas" es tal, que se ha reflejado en su actividad legislativa. Por ejemplo, en abril de 2002, se presentaron en el Congreso de los Diputados tres Proposiciones de Ley a petición de PSOE, IU e Iniciativa per Catalunya-Els Verds para reformar la Ley Orgánica de Régimen Electoral de 1985 (LOREG) en aras a equilibrar la presencia de hombres y mujeres en las listas electorales. Pero también a la hora de elaborar la mismas, al menos oficialmente, porque como señalaba Eduardo Zaplana, cuando era el Portavoz del Grupo Parlamentario Popular en el Congreso de los Diputados, "los partidos, al confeccionar las listas, tendrán en cuenta la capacidad, competencia y dedicación de cada candidato, y la asunción por el mismo de los principios éticos y políticos que defiendan éstos, quedando excluida toda discriminación por razón de nacimiento, raza, sexo, orientación sexual, edad, religión, discapacidad o cualquier otra condición o circunstancia personal o social [...]. Establecer o imponer cualquier tipo de techo para lograr la efectiva igualdad de oportunidades, no supone otra cosa que minusvalorar las capacidades individuales y personales de todo ciudadano". Pero ahí, no queda la cosa, las propias mujeres del PP son las primeras en manifestarse en contra de este tipo de medidas, cuando realizan afirmaciones como que "las mujeres del PP no queremos estar en unas listas electorales por cuotas ni por cremalleras, porque nos parece humillante y un atentando a nuestra dignidad, porque creemos que estamos aquí porque en su día nuestro partido pensó que éramos las mejores". Pero esto no significa que este partido no haya establecido ningún tipo de estrategia en este sentido.

En el PSOE, en cambio, la estrategia es completamente distinta, al convertirse en el primer partido español en apostar por un sistema de "cuotas", después también se sumaría a este tipo de estrategia la coalición IU. Fue en 1988, cuando los socialistas introdujeron en su normativa la primera medida a favor de la discriminación positiva al establecer la cuota femenina del $25 \%$. Poco después, en 1994 garantizaron que cuando en un ámbito territorial la afiliación femenina fuera mayor del $25 \%$ la reserva de puestos sería cinco puntos superior a dicho porcentaje. En 1997 dieron un paso más y se exigió el cumplimiento de un principio de democracia paritaria basado en el criterio de que ningún grupo de sexo tenga una presencia menor del $40 \%$ ni mayor $60 \%$. Pero lo realmente importante de esta medida no son los porcentajes que se establecen sino que éstos se tienen que aplicar no sólo en el conjunto global de las listas electorales sino dentro de los puestos que razonablemente cuentan con posibilidades de ser electos. Pero tal vez, la acción con mayor impacto y repercusión haya sido la de Ley de Igualdad propuesta y aprobada por el Gobierno socialista de José Luis Rodríguez Zapatero. Dicha Ley se articula sobre la base de favorecer el acceso efectivo de la mujer al empleo, la protección de la maternidad y la paternidad, la conciliación de la vida personal y laboral, la prevención y protección frente al acoso, y medidas que favorecen la paridad tanto en las listas electorales como en el mundo laboral, es decir, que no sólo se vela por los aspectos cuantitativos sino también por los cualitativos, que a la larga son más importantes que los primeros, al facilitar realmente la incorporación de la mujer. 


\section{EVALUACIÓN DEL GRADO DE PARTICIPACIÓN FEMENINA EN LOS ÓRGANOS NACIONALES DEL PARTIDO}

Hasta este momento hemos estado hablando de las estrategias que han desarrollado los partidos políticos para favorecer la igualdad en sus organizaciones. Pero ahora vamos evaluar realmente la repercusión que éstas han teniendo en la propia estructura organizativa de los mismos. Por eso llegado a este punto deberíamos realizarnos preguntas tales como: ¿la incorporación de la mujer a la vida política ha sido más efectiva en los partidos que han establecido un sistema de cuotas o en los que no? y ¿la participación en política de las mujeres es real o simplemente simbólica? Para contestarlas analizaremos la composición en los órganos que a priori rigen la vida del partido entre congresos, que en el caso del PSOE será el Comité Federal (CF) y la Comisión Ejecutiva Federal (CEF), y en el del PP el Comité Ejecutivo Nacional (CEF) y la Junta Directiva Nacional (JDN).

El reflejo de las disposiciones estatutarias socialistas a favor de la igualdad se puede comprobar en los integrantes de los dos órganos de decisión del partido, la Comisión Ejecutiva Federal (CEF) y el Comité Federal (CF). Así, mientras que en 1990, el 21,20\% de los miembros de la CEF eran mujeres, en 1994 este porcentaje se elevó en 10,2 puntos, en 1997 en 4,96 puntos, para decaer en 2004 en 1,7 puntos, y volver a repuntar en el 2008 al superar por fin la barrera del 50\% (ver tabla 2). En el CF también se ha incrementado la presencia femenina, ya que mientras que en 1988 éstas sólo representaban el 13,4\% sobre el total de sus miembros, en 1990 su participación creció en 7,1 puntos, en 1994 en 7,7, en 1997 en 11, en 2000 se mantuvo en los mismos números para volver a crecer en 4,7 puntos en 2004 (ver tabla 2). Otro dato importante en relación con este tema han sido las secretarías que han venido ocupando las mujeres dentro de la CEF, entre las que cabe mencionar las siguientes: Secretaría de Organización, Carmen García Bloise (1979-1981) y Leire Pajín (2008-..); Secretaría de Relaciones Internacionales, Elena Flores (1984-1990) y Trinidad Jiménez (2000-2003); Secretaría de Participación de la Mujer, Carmen García Bloise (1990-1994), Carmen Hermosín Bono (1994-1997), y Micaela Navarro (1997-..); Secretaría de Emigración, Carmen García Bloise (1990-1994); Secretaría de Relación con la Sociedad, Carmen Cerdeira (19972000) y Leire Pajín Iraola (2000-..); Secretaría de Bienestar Social, Clementina Díez Baldeón (1997-2000); Secretaría de Comunicación, Ana Miranda (1988-1990); Secretaría de Políticas Sociales y Migratorias, Consuelo Rumí Ibáñez (2000-..); Secretaría de Medio Ambiente y Ordenación Territorial, Cristina Carbona (2000-..); Secretaría de Educación, Universidad, Cultura e Investigación, Carmen Chacón (2000-..); Secretaría de Consumidores y Usuarios, Isabel Pozuelo Meño (2000-..). Por tanto, las mujeres han ostentado tanto secretarías de máxima relevancia (Secretaría de Organización, Secretaría de Relaciones Internacionales, etc.) como otras que son más bien simbólicas en función de la importancia de las competencias atribuidas (Secretaría de Consumidores y Usuarios, Secretaría de Bienestar Social, Secretaría de Emigración, etc). Aunque nunca han desempeñado las funciones que competen a la vicesecretaría general, o la propia secretaría general, si han llegado a la Secretaría de Organización, que es el tercer puesto en importancia del partido.

Tabla 2. Porcentaje de participación femenina en el CF y la CEF (1979/2004)

\begin{tabular}{|c|c|c|c|c|c|c|c|c|c|c|}
\hline Años & $\mathbf{1 9 7 9}$ & $\mathbf{1 9 8 1}$ & $\mathbf{1 9 8 4}$ & $\mathbf{1 9 8 8}$ & $\mathbf{1 9 9 0}$ & $\mathbf{1 9 9 4}$ & $\mathbf{1 9 9 7}$ & $\mathbf{2 0 0 0}$ & $\mathbf{2 0 0 4}$ & $\mathbf{2 0 0 8}$ \\
\hline CF & 3,22 & ---- & 5,50 & 13,4 & 20,5 & 28,20 & 40,8 & 40.8 & 45,5 & --- \\
\hline CEF & 8 & 12 & 17,60 & 26,10 & 21,20 & 31,40 & 42,4 & 40 & 40 & 51,56 \\
\hline
\end{tabular}

Fuente: Elaboración propia 
No obstante, los socialistas, pese haberse pronunciado a favor de la democracia paritaria, en la mayoría de las ocasiones los órganos de dirección no han respetado los principios normativos, sólo lo han hecho a partir de 1997 y sólo en el 2008 las mujeres han llegado a representar el $50 \%$ de la Comisión Ejecutiva Federal. Aunque también es cierto que su presencia en dichos órganos se ha ido incrementando paulatinamente, es más podríamos decir que se ha triplicado.

Tabla 3. Porcentajes de participación femenina en la JDN (1978/1999)

\begin{tabular}{|l|c|c|c|c|c|c|c|c|c|c|c|c|}
\cline { 2 - 12 } \multicolumn{1}{c|}{} & 1978 & 1979 & 1981 & 1982 & 1984 & $\mathbf{1 9 8 6}$ & $\mathbf{1 9 8 7}$ & $\mathbf{1 9 8 9}$ & $\mathbf{1 9 9 0}$ & $\mathbf{1 9 9 3}$ & $\mathbf{1 9 9 6}$ & $\mathbf{1 9 9 9}$ \\
\hline PUAP & 5 & & & & & & & & & & & \\
\hline FAP & & & & & & & & & & & & \\
\hline AP & & & & & & & & & & & & \\
\hline PP & & & & & & & & & & 11 & 14 & 15 \\
\hline
\end{tabular}

Fuente: (Ruiz Jiménez, 2002: 1)

En los estatutos del PP, como ya hemos explicado, no se hace ninguna referencia específica a la participación de las mujeres, únicamente se alude al derecho que tienen los afiliados para concurrir como candidatos a formar parte de los órganos de gobierno o de las candidaturas que el partido presenta en los diferentes comicios. No existen cuotas, aunque desde la llegada de José María Aznar a la presidencia del partido, se ha dado prioridad a que los órganos de dirección vayan incorporando mujeres, todavía son irrelevantes si los comparamos con el porcentaje que representan los hombres en los mismos, o en otros partidos, como en el PSOE. Téngase en cuenta que en el máximo órgano ejecutivo, las mujeres socialistas se sitúan en casi 22 puntos por encima de las populares.

Tabla 4. Porcentaje de participación femenina en el CEN (1978/2004)

\begin{tabular}{|l|c|c|c|c|c|c|c|c|c|c|c|c|c|c|}
\cline { 2 - 16 } \multicolumn{1}{c|}{} & 1978 & $\mathbf{1 9 7 9}$ & $\mathbf{1 9 8 1}$ & $\mathbf{1 9 8 2}$ & $\mathbf{1 9 8 4}$ & $\mathbf{1 9 8 6}$ & $\mathbf{1 9 8 7}$ & $\mathbf{1 9 8 9}$ & $\mathbf{1 9 9 0}$ & $\mathbf{1 9 9 3}$ & $\mathbf{1 9 9 6}$ & $\mathbf{1 9 9 9}$ & $\mathbf{2 0 0 4}$ & $\mathbf{2 0 0 8}$ \\
\hline PUAP & & 12 & 7 & 11 & 5 & 7 & & & & & & & & \\
\hline FAP & & 12 & 4 & & 8 & 13 & & & & & & & & \\
\hline AP & & & & & & & 7 & & & & & & & \\
\hline PP & & & & & & & & 10 & 18 & 18 & 12 & 22 & 26.8 & 29.9 \\
\hline
\end{tabular}

Fuente: (Ruiz Jiménez, 2002)

En todo caso, como hemos mencionado, en el PP los órganos de dirección son la JDN y el CEN. En el primero, las mujeres representan el 15\% y en el segundo su presencia es algo más elevada al alcanzar una cifra del 29,9\%. Porcentajes que todavía no se acercan a los que presentan los órganos de dirección socialista, pero que han supuesto un enorme crecimiento de las mujeres populares en los mismos, ya que durante los años ochenta éstos nunca fueron superiores al 13\%. Sólo desde la llegada de Aznar a la presidencia del partido se ha producido un incremento gradual hasta alcanzar su punto más álgido en 2008. El problema es que pese a aumentar su presencia en los órganos de dirección, las mujeres continúan desempeñando puestos y funciones secundarias, dado que el liderazgo femenino es poco probable y cuando se produce está constreñido a las áreas diseñadas por la oligarquía masculina del partido. Por ejemplo, en la ejecutiva saliente del XIII Congreso Nacional, Ana Mato y Mercedes de la Merced, son nombradas Coordinadora de Participación y Coordinadora de Formación, respectivamente. La figura de los coordinadores está sujeta a la secretaría general, que en toda su historia ha sido ostentada por hombres, con la única excepción María Dolores de Cospedal. Aunque también es cierto que en la penúltima 
ejecutiva, la del XV Congreso Nacional (2004), ya se empezó a invertir esta tendencia, y las mujeres comenzaron a desempeñar puestos de mayor importancia dentro del CEN. Así, por ejemplo, Sandra Moneo ha alcanzado una de las seis secretarías, y Soroya Sáenz de Santamaría y Elvira Rodríguez Herrer se han alzado con dos secretarías ejecutivas más importantes, la de política autonómica y local, y de la política económica y empleo, respectivamente. Pero será en el XVI Congreso Nacional (2008) cuando por primera una mujer ocupa la Secretaría General del partido y la vicesecretaría general de organización y electoral, que son dos de los puestos claves de esta organización.

\section{EVALUACIÓN DEL GRADO DE PARTICIPACIÓN FEMENINA EN LOS CARGOS DE REPRESENTACIÓN NACIONAL}

Si nos volviéramos a realizar las mismas preguntas que en el apartado anterior pero esta vez refiriéndonos a los cargos de representación tendríamos que contestar de la siguiente manera. A nivel nacional, el ejecutivo que ha contado con mayor número de mujeres es el de José Luís Rodríguez Zapatero (Presidente del gobierno desde 2004). El primero de sus gobiernos contó con ocho mujeres $(50 \%)$ para ejercer labores ministeriales y en el segundo fueron $9(52,94 \%)$, por tanto, podemos decir que la paridad del 50\% impuesta por el PSOE tiene un efecto sumamente positivo para la incorporación de las mujeres en la vida política. Además, si durante su primer mandato las carteras ministeriales ocupadas por mujeres, si exceptuamos la Vicepresidencia Primera, eran de segundo orden, durante la segunda legislatura la cosa cambió de forma considerable al situar a mujeres al frente del ministerio de Defensa, Fomento, o Ciencia y Tecnología. Pero también es cierto, que las mujeres han tenido un peso significativo en el PP sin disponer un sistema de cuotas para ello. En los gobiernos presididos por José María Aznar (Presidente del Gobierno por el PP desde 1996 a 2004) el papel de las mujeres ha sido importante. En el primer ejecutivo participaron cuatro mujeres $(26,97 \%)$ mientras que en el segundo esta cifra se elevó a siete $(17,56 \%)$.

Pero qué sucedería si nos centráramos en los restantes escalones de la estructura políticoadministrativa. Comprobaríamos que la paridad no se cumple en el resto de los puestos de responsabilidad del Estado, pues en ellos, las mujeres han quedado relegadas a un segundo plano ocupando tres de cada diez altos cargos. Además, pese a todo, la mujer todavía no ha conseguido abrirse camino en áreas tradicionalmente reservadas a los hombres. Por ejemplo, sorprende que sólo disfruten de una representación meramente testimonial en los puestos de responsabilidad del ministerio de Defensa, donde están adscritos los tres ejércitos. Tan sólo el Instituto Social y el Instituto de Vivienda de las Fuerzas Armadas, que tienen categoría de dirección general, dependen de mujeres. En el ministerio de Interior y de Exteriores los porcentajes de mujeres también se hallan lejos de los parámetros de paridad. Lo mismo sucede, en el ministerio de Fomento y en el de Medio Ambiente, y eso a pesar que están dirigidos por mujeres, ya que en éstos la representación femenina se reduce al 14,5\% en el primero, y al $27,5 \%$ en el segundo. Los ministerios que se encuentran a medio camino de la paridad son los de Economía (26,1\%), Industria (27\%), Administraciones Públicas (29\%), Educación (31\%), Sanidad (32,6\%), y Trabajo y Asuntos Sociales (33,3\%). Y sólo en el ministerio de Cultura (54\%) y en el de Vivienda (52\%) se alcanza la total paridad, cerca de ella se quedan el ministerio de Justicia (41\%) y el de Vicepresidencia (38\%). 


\section{a) El papel de las mujeres en las Cortes Generales}

En otro orden de cosas, habría que decir que en el año 2004 España estaba situada en el octavo puesto en cuanto al número de mujeres en sus parlamentos, por delante de países como Italia, Portugal, Reino Unido, Francia, Grecia, e Irlanda, pero todavía lejos de las cifras que alcanzan los países del norte de Europa. Y esto se debe en gran medida a que la composición de las instituciones representativas viene determinada por el proceso de selección que los partidos políticos establecen para elaborar sus listas electorales. La inclusión de mujeres en las mismas va a depender de las decisiones que adopten a este respecto las élites que dominan las organizaciones. Por tanto, pese a tener la necesidad de una cierta presencia femenina para alcanzar una representación y una legitimidad social, el número de mujeres en ellas todavía es escaso, y sus posibilidades de éxito están determinadas por una clase dominante integrada mayoritariamente por varones que se muestran poco dispuestos a situar a mujeres en los primeros puestos cuando las listas son reducidas (García de León, 1991:49). Pero además, normalmente, las mujeres electas para cargos públicos han ocupado áreas de acción de segundo orden. La razón, puede obedecer, en primer lugar, a la falta de ambición mostrada por las mujeres a este respecto. Sin embargo, hay que tener en cuenta que este rasgo en términos generales posee unas connotaciones positivas para los hombres pero negativas para las mujeres (Uriarte y Elizondo, 1997:67). En segundo lugar, a las barreras que tienen para desempeñar cargos de cierta relevancia, como son las cargas familiares o las que la propia organización establece.

Otro dato que debemos tener en consideración es la influencia que ejerce cada sistema político en la participación política de las mujeres. Los estudios que se han desarrollado sobre las mujeres y los partidos políticos han concluido que los tres factores que determinan la presencia femenina son: la estructura de la candidatura (personal o lista), la magnitud del distrito (número de escaños por los que se compiten) y el grado de proporcionalidad del sistema (proporcional o mayoritario) (Uriarte y Elizondo, 1997:98/99). Teniendo presente esto, podemos decir que los sistemas multipartidistas ofrecen mayores posibilidades para las mujeres que los bipartidistas; que los sistemas plurinominales son más favorables para la elección de mujeres que los uninominales; y que los sistemas donde los escaños se asignan proporcionalmente, el porcentaje de mujeres suele ser superior al de los sistemas mayoritarios. Por tanto, España cuenta con todos los factores que favorecen y facilitan la integración de las mujeres en las listas electorales, dado que su sistema político se caracteriza por ser multipartidistas, plurinominal y proporcional.

Tabla 5. Porcentaje de candidatas. Listas electorales del PSOE a las Cortes Generales

\begin{tabular}{|c|c|c|c|c|c|c|c|}
\hline Congreso & $\mathbf{1 9 7 9}$ & $\mathbf{1 9 8 2}$ & $\mathbf{1 9 8 6}$ & $\mathbf{1 9 8 9}$ & $\mathbf{1 9 9 3}$ & $\mathbf{1 9 9 6}$ & $\mathbf{2 0 0 0}$ \\
\hline$\%$ & 10 & 10 & 14 & 29 & 29 & 38 & 47 \\
\hline$(1 / 2)$ & $(52)$ & $(41)$ & $(29)$ & $(39)$ & $(42)$ & $(49)$ & $(51)$ \\
\hline SENADO & $\mathbf{1 9 7 9}$ & $\mathbf{1 9 8 2}$ & $\mathbf{1 9 8 6}$ & $\mathbf{1 9 8 9}$ & $\mathbf{1 9 9 3}$ & $\mathbf{1 9 9 6}$ & $\mathbf{2 0 0 0}$ \\
\hline$\%$ & 3 & 6 & 7 & 20 & 25 & 36 & 37 \\
\hline
\end{tabular}

Candidaturas proclamadas; \% de mujeres sobre el total de candidatos de lista Fuente: (Ruiz Jiménez, 2002: 98)

Pese a todo ello, la presencia femenina en las Cortes Generales sólo empieza a tener cierta relevancia a partir de 1989, aunque todavía se encuentra alejada de los postulados de democracia paritaria que han sido, por ejemplo, defendidos por los socialistas (Sánchez, 2000). No obstante, en el PSOE, entre 1993 y el 2000 se ha producido un incremento 
porcentual de 18 puntos en el Congreso y de 12 en el Senado, aumento que puede deberse a las medidas introducidas en los estatutos y reglamentos para paliar la escasez de mujeres en estos ámbitos representativos (ver tabla 5). Sin embargo, este crecimiento en las candidaturas de ambas cámaras no ha visto correlación en su presencia en las mismas. Porque una cosa es que se incluyan en las listas y otra muy distinta que sean situadas en posiciones donde su elección este asegurada. Lo que ha ocurrido, es que la cuota del $25 \%$, primero y la del $40 \%$ después, se ha aplicado a lo largo y ancho de toda la lista, por lo tanto, son muy pocas las mujeres que se sitúan en los puestos de salida, lo que conlleva una escasa representación femenina en las cámaras parlamentarias. Además, que una medida se aplique no implica que tenga un efecto inmediato, sino que más bien éste suele producirse pasado un tiempo.

Tabla 6. Porcentaje de las mujeres del PSOE en las Cortes Generales

\begin{tabular}{|c|c|c|c|c|c|c|c|c|c|c|}
\hline Congreso & $\begin{array}{c}1977 / \\
79\end{array}$ & $\begin{array}{c}1979 / \\
82\end{array}$ & $\begin{array}{c}1982 / \\
86\end{array}$ & $\begin{array}{c}1986 / \\
89\end{array}$ & $\begin{array}{c}1989 / \\
93 \\
\end{array}$ & $\begin{array}{c}1993 / \\
96\end{array}$ & $\begin{array}{c}1996 / \\
00\end{array}$ & $\begin{array}{c}2000 / \\
04\end{array}$ & $\begin{array}{c}2004 / \\
08\end{array}$ & $\begin{array}{c}2008 / \\
\ldots .\end{array}$ \\
\hline Mujeres & ---- & ---- & 14 & 13 & 30 & 28 & 39 & 46 & ---- & --- \\
\hline$\%$ & 9 & 5 & 6,93 & 7,07 & 17,14 & 17,61 & 27,86 & 36,80 & 46,30 & 43,20 \\
\hline Senado & $\begin{array}{c}1977 / \\
79 \\
\end{array}$ & $\begin{array}{c}1979 / \\
82 \\
\end{array}$ & $\begin{array}{c}1982 / \\
86 \\
\end{array}$ & $\begin{array}{c}1986 / \\
89 \\
\end{array}$ & $\begin{array}{c}1989 / \\
93 \\
\end{array}$ & $\begin{array}{c}\text { 1993/ } \\
96\end{array}$ & $\begin{array}{c}1996 / \\
00\end{array}$ & $\begin{array}{c}2000 / \\
04\end{array}$ & $\begin{array}{c}2004 / \\
08 \\
\end{array}$ & $\begin{array}{c}2008 / \\
\ldots\end{array}$ \\
\hline Mujeres & ---- & ---- & 10 & 12 & 18 & 21 & 22 & 12 & ---- & --- \\
\hline$\%$ & 3 & 3 & 6,37 & 8,05 & 14,06 & 17,95 & 22,92 & 17,39 & 27,08 & 29 \\
\hline
\end{tabular}

Fuente: Instituto de la Mujer

Solamente en el Congreso, en la sexta legislatura (1996/2000) y en la séptima (2000/2004), la diferencia entre candidatura y representación decayó en 10 puntos, lo que significa que la inclusión de mujeres en las listas electorales deja de ser simbólica para convertirse en una realidad factible de representación. Aunque eso sí, en el Senado se ha producido un proceso inverso, cada vez es mayor la diferencia porcentual entre la candidatura y la representación, alcanzando los 19 puntos en la séptima legislatura (2000/2004) (Ver tabla 6). Y como consecuencia de ello, los socialistas han terminado por inclinarse en la mayoría de las circunscripciones por lo que se ha denominado "la cremallera", es decir, hombre-mujer-hombre-mujer (Cernuda, 2000: 155), para de esta forma dar cumplimiento a las disposiciones estatutarias, con lo cual ningún sexo tenga una presencia menor al $40 \%$ ni mayor al $60 \%$. Disposición reglamentaria que, por otra parte, sólo se ha cumplido a partir de la octava legislatura $(2004 / \ldots)$ donde las mujeres se sitúan en $46,30 \%$ y los hombres en $53,7 \%$. Por tanto, se podría decir que la ejecutiva socialista ha sido bastante flexible a la hora de aplicar las medidas reglamentarias que ella misma ha elaborado y aprobado, porque tan sólo en dos ocasiones han alcanzado la tan deseada igualdad representativa. Por el contrario, en el Senado la situación es todavía peor. En ninguna legislatura se ha llegado a la tan ansiada igualdad paritaria, y sólo a partir de 2004 se ha logrado superar la barrera del $25 \%$.

En todo caso, no son pocos los avances que se han efectuado en este sentido, habida cuenta que hasta 1989 el número de ellas era prácticamente irrisorio al no superar en ninguno de los casos las trece diputadas, más aún cuando esa época coincide con la de mayor respaldo electoral conseguido por el partido. Cabe recordar aquí, por ejemplo, que 
en 1982 el PSOE obtuvo el mejor resultado electoral que ha logrado cualquier partido en España con 202 diputados, lo que atribuye mayor valor a los porcentajes de $36,80 \%$ que arrojan la séptima legislatura (2000/2004) cuando el número de diputados se situó en 125 . No obstante, la Ley de la Igualdad todavía no ha logrado imponer una representación en las Cortes del 50\%, como en un principio pretendía. En el mejor de los casos, se han logrado unos porcentajes del $46 \%$ en la cámara baja y del 29 en la cámara alta.

Tabla 7. Porcentaje de candidatas en las listas electorales de AP/PP a las Cortes Generales.

\begin{tabular}{|c|c|c|c|c|c|c|c|}
\hline Congreso & $\mathbf{1 9 7 9}$ & $\mathbf{1 9 8 2}$ & $\mathbf{1 9 8 6}$ & $\mathbf{1 9 8 9}$ & $\mathbf{1 9 9 3}$ & $\mathbf{1 9 9 6}$ & $\mathbf{2 0 0 0}$ \\
\hline$\%$ & 14 & 11 & 13 & 17 & 22 & 26 & 34 \\
\hline$(1 / 2)$ & $(47)$ & $(34)$ & $(34)$ & $(38)$ & $(39)$ & $(37)$ & $(46)$ \\
\hline SENADO & $\mathbf{1 9 7 9}$ & $\mathbf{1 9 8 2}$ & $\mathbf{1 9 8 6}$ & $\mathbf{1 9 8 9}$ & $\mathbf{1 9 9 3}$ & $\mathbf{1 9 9 6}$ & $\mathbf{2 0 0 0}$ \\
\hline$\%$ & 4 & 4 & 8 & 14 & 15 & 17 & 34 \\
\hline
\end{tabular}

* Candidaturas proclamadas; \% de mujeres sobre el total de candidatos de lista Fuente: (Ruiz Jiménez, 2002: 98)

En lo referente al PP, podríamos decir que parece haberse dado cuenta del cambio que se está produciendo en la preferencia partidista de las mujeres, y que está favoreciendo a los socialistas. El hecho de que el partido no sea capaz de llegar a este sector de la población, se considera, en términos generales, como un gran problema. De ahí que el objetivo que se marca el partido sea mejorar la percepción y la valoración del electorado femenino respecto a su organización (Ruiz Jiménez, 2002: 333), sin por ello caer en el sistema de cuotas como el partido socialista. Lo curioso es que la presencia de mujeres es más elevada en los órganos internos del partido que en las Cortes Generales, cuando la repercusión mediática que consiguen las segundas es mucho menor. Parece una contradicción, pero la realidad es esa y no otra. En cualquier caso, las mujeres tienen una mayor presencia en las candidaturas del partido en el Congreso y en el Senado. En 1989, incrementan en cuatro y seis puntos sus porcentajes en las listas del partido. En 1993 lo hacen en cinco puntos en el Congreso y uno en el Senado, en 1996 son cuatro en el primero y dos en el segundo, y en 2000, son ocho y diecisiete, respectivamente. Esto es igual a decir que en 1986 el porcentaje de mujeres en las listas electorales ha crecido en 21 puntos en el Congreso y 26 en el Senado. No está nada mal si tenemos en cuenta que en un principio, en ninguno de los dos casos, llegaban a superar la barrera del 14\%. Aunque todavía son números relativamente pobres al estar muy por debajo de los que presenta el partido socialista, en concreto 13 puntos de distancia entre uno y otra formación. Pero también hay que mencionar que, al igual que ocurre en el PSOE, hay cierta disintonía entre las candidaturas femeninas y los cargos electos que éstas tienen, aunque en menor medida que en el caso socialista, al mantener una media de 8,6 en el Congreso y de 5,5 en el Senado, mientras que los socialistas sitúan la misma en 11,9 y en 11,4, respectivamente. Lo que significa que los populares colocan a un mayor número de mujeres en los puestos que tiene mayores posibilidad para salir elegidas, por tanto, sus listas cuentan con menos mujeres pero mejor posicionadas.

Respecto a su representación en las Cortes Generales, cabría señalar que, a diferencia de lo que sucede en el PSOE, las mujeres se encuentran representadas en ambas cámaras prácticamente con los mismos porcentajes. Es más, en algunas legislaturas, logran mayor presencia en el Senado que en el Congreso. Lo que indica que los populares ceden más puestos en la lista de la cámara alta, que es precisamente la que disfruta de menor poder 
real, que en la cámara baja que es donde se adoptan realmente las decisiones que regirán el país.

Tabla 8. Porcentaje de las mujeres de AP/PP en las Cortes Generales

\begin{tabular}{|c|c|c|c|c|c|c|c|c|c|c|}
\hline Congreso & $\begin{array}{c}1977 \\
/ 79\end{array}$ & $\begin{array}{c}1979 \\
/ 82\end{array}$ & $\begin{array}{c}1982 \\
/ 86\end{array}$ & $\begin{array}{c}1986 \\
/ 89\end{array}$ & $\begin{array}{c}1989 \\
/ 93\end{array}$ & $\begin{array}{c}1993 \\
/ 96\end{array}$ & $\begin{array}{c}1996 \\
/ 00\end{array}$ & $\begin{array}{c}2000 \\
/ 04\end{array}$ & $\begin{array}{c}2004 \\
/ 08\end{array}$ & $\begin{array}{c}2008 \\
/ \ldots\end{array}$ \\
\hline Mujeres & & & 1 & 5 & 11 & 21 & 22 & 46 & 42 & ---- \\
\hline$\%$ & 6 & 11 & 0.9 & 5.9 & 10.3 & 14.8 & 14.2 & 25.1 & 28.3 & 29.87 \\
\hline SENADO & $\begin{array}{c}1977 \\
/ 79\end{array}$ & $\begin{array}{c}1979 \\
/ 82 \\
\end{array}$ & $\begin{array}{c}1982 \\
/ 86\end{array}$ & $\begin{array}{c}1986 \\
/ 89 \\
\end{array}$ & $\begin{array}{c}1989 \\
/ 93 \\
\end{array}$ & $\begin{array}{c}1993 \\
/ 96 \\
\end{array}$ & $\begin{array}{c}1996 \\
/ 00\end{array}$ & $\begin{array}{c}2000 \\
/ 04 \\
\end{array}$ & $\begin{array}{c}2004 \\
/ 08\end{array}$ & $\begin{array}{c}2008 \\
/ \ldots\end{array}$ \\
\hline Mujeres & & & 1 & 2 & 7 & 10 & 14 & 46 & 30 & --- \\
\hline$\%$ & & & 1.4 & 3.1 & 7.7 & 9.4 & 10.5 & 30.8 & 24.6 & 29.17 \\
\hline
\end{tabular}

Fuente: Instituto de la Mujer

No obstante, su representación en las Cortes Generales se ha visto incrementada en unos números realmente importantes, en concreto han sido 22,87 puntos en el Congreso y 27,77 en el Senado. Pero si los comparamos con las cifras que arrojan los grupos parlamentarios socialistas el crecimiento experimentado por las mujeres populares se vería medio ensombrecido, al situarse 11,33 puntos por debajo en el Congreso, aunque en el Senado superaría a las socialistas en 1,77 puntos. Por tanto, según los datos que nos han proporcionado estas tablas, podemos concluir que los sistemas de cuotas a priori han resultado ser más efectivos para garantizar la inclusión de las mujeres en las cámaras de representación nacional, dada cuenta que el partido que se ha decantado por ellas, el socialista, cuenta con un mayor número de mujeres en sus grupos parlamentarios que los que no lo han hecho, los populares.

En todo caso, viendo la representación que las mujeres de ambos partidos tienen en las Cortes Generales, cabe advertir que la Ley de Igualdad apenas ha tenido repercusión. Si miramos los datos podríamos llegar a afirmar que no se ha producido ningún avance, incluso, es más, en el caso del PSOE se podría hablar de un ligero retroceso. Todo porque con ley o sin ella, el primer puesto de la lista sigue estando reservado, mayoritariamente, a los hombres, ya que en las últimas elecciones generales más del $70 \%$ de las cabezas de listas de ambos partidos eran candidatos. Mientras que las mujeres han quedado relegadas, en el mejor de los casos, al segundo puesto, ya que en muchos otros ni si quieran llegan a ocupar ni el primer ni el segundo puesto, y la ley se extiende a lo largo de toda la candidatura. Pero incluso si fuéramos más allá, también veríamos como la Ley no se cumple en ningún otro ámbito. Así, el poder en las empresas del Ibex 35 continúa en manos de los hombres, incluso en muchos consejos de administración no hay ni una sola mujer. De los 512 consejeros que existen en esas compañías, sólo 26 son mujeres, representando apenas el $5 \%$, los mismos números que cuando en marzo de 2004 se aprobó la ley de igualdad. Por tanto, la realidad está muy alejada del objetivo que persigue la normativa. Para cumplirlo, deberían incorporarse cerca de 200 mujeres a los órganos de dirección de las empresas del Ibex 35.

Otra cuestión que nos ayuda a valorar el efecto del sistema de cuotas es el grado de permanencia de las diputadas. En primer lugar, son las mujeres del PP las que mantienen mayores porcentajes de permanencia, su media se sitúa en $65,3 \%$. Lo que nos indica dos cosas: 1) el sistema de cuotas posibilita una mayor circulación de los cargos institucionales 
femeninos, porque sino las mujeres populares deberían contar con porcentajes mayores que las socialistas, habida cuenta que si por algo se caracterizó la presidencia de Aznar fue por una renovación de su élite, y 2) el sistema de cuotas favorece una mayor presencia de mujeres pero no implica que desempeñen funciones más relevantes, porque sino seguramente no se habría producido tanta movilidad.

Tabla 9. Diputadas que repite legislatura (1986/2004)

\begin{tabular}{|l|c|c|c|c|c|c|c|c|c|}
\cline { 2 - 10 } \multicolumn{1}{c|}{} & $\mathbf{1 9 7 7 / 7 9}$ & $\mathbf{1 9 7 9 / 8 2}$ & $\mathbf{1 9 8 2 / 8 6}$ & $\mathbf{1 9 8 6 / 8 9}$ & $\mathbf{1 9 8 9 / 9 3}$ & $\mathbf{1 9 9 3 / 9 6}$ & $\mathbf{1 9 9 6 / 0 0}$ & $\mathbf{2 0 0 0 / 0 4}$ & Media \\
\hline PSOE & $44,4(4)$ & $75(6)$ & $52,6(10)$ & $73,7(14)$ & $56,8(21)$ & $39,4(13)$ & $46,8(22)$ & $44,6(25)$ & 54,2 \\
\hline PP & $100(1)$ & $100(1)$ & $25(1)$ & $46,2(6)$ & $75(9)$ & $73,9(17)$ & $67,7(21)$ & $34,8(23)$ & 65,3 \\
\hline
\end{tabular}

Fuente: Elaboración propia, en base a los datos del Archivo del Congreso de los Diputados

En segundo lugar, habría que indicar que en el PP, a partir de 2004, se produce un fenómeno observado en el PSOE desde 1993, aunque de manera menos acentuada: a medida que aumenta la presencia de las mujeres en el Grupo Parlamentario, éstas sufren sistemáticamente una mayor renovación que los hombres (Verge, 2006: 188). En tercer lugar, los altos índices de renovación de las diputadas socialistas pueden deberse a tres causas distintas: 1) a los cambios de liderazgo en el PSOE (abandono de la secretaria de Felipe González en 1997, la llegada de Joaquín Almunia a la Secretaría General en 2000 y de José Luís Rodríguez Zapatero en el 2004), 2) a las luchas entre las distintas familias a la hora de elaborar las listas electorales, y 3) a la propia política de cuotas que ha llevado a contar con mujeres más que por su valía por la de cubrir el expediente numérico que marcan los estatutos del partido. En todo caso, parece que para garantizar la permanencia de los cargos electos femeninos, el sistema de cuotas no ha supuesto un efecto positivo como sucedía con las anteriores cuestiones.

\section{b) La presencia de mujeres en los altos cargos de la Administración Pública}

Por otra parte, también hemos tenido en cuenta como los sistemas de cuotas han incidido en la Administración Pública. Según los datos de la Tabla 10, la participación de las mujeres en este ámbito de poder, el de los altos cargos, es más bien reducida, sólo en el caso de las carteras ministeriales $\mathrm{y}$, últimamente, en las direcciones generales y subsecretarias están consiguiendo tener cierta presencia. En primer lugar, respecto a los ministerios habría que decir, que pese a que es cierto, como hemos explicado, que en los gobiernos de José María Aznar las mujeres han jugado un papel destacado, en ningún momento de nuestra historia democrática, han llegado a ocupar tantas carteras ministeriales como en el segundo ejecutivo de José Luis Rodríguez Zapatero. Además, habría que diferenciar otro hecho, en los gobiernos populares las ministras han venido a desempeñar funciones básicas, como se pone de manifiesto en la tabla 11, por contra, las mujeres socialistas que se han encontrado integradas en los gobiernos del PSOE han desarrollado todo tipo de funciones, por tanto, han dispuesto a priori de poder.

En segundo lugar, las direcciones generales son el siguiente nivel en el que las mujeres se han encontrado con mayores posibilidades para participar en la vida política. En 1995, éstas representaban el 13.75\%, en 1996, coincidiendo con el primer gobierno del PP, esta cifra desciende en 2,26 puntos, para luego repuntar en el 2000 y, de nuevo, en el 2004. 
No obstante, la media de los gobiernos socialistas $(20,47)$ es más elevada que la de los gobiernos populares $(14,9)$.

Tabla 10. Altos cargos en la Administración Pública

\begin{tabular}{|l|c|c|c|c|c|c|c|c|c|}
\cline { 2 - 10 } \multicolumn{1}{c|}{} & $\mathbf{1 9 9 5}$ & $\mathbf{1 9 9 6}$ & $\mathbf{1 9 9 9}$ & $\mathbf{2 0 0 0}$ & $\mathbf{2 0 0 1}$ & $\mathbf{2 0 0 2}$ & $\mathbf{2 0 0 4}$ & $\mathbf{2 0 0 6}$ & $\mathbf{2 0 0 7}$ \\
\hline Ministras & 20 & 28.57 & 14.29 & 18.75 & 18.75 & 18.75 & 50 & 44.44 & 43.75 \\
\hline Secretaria de Estado & 25 & & 4.17 & 3.85 & 3.85 & 3.70 & 12 & 21.74 & 20 \\
\hline Subsecretarias & 3.09 & 7.02 & 8.20 & 13.70 & 18.84 & 22.06 & 22.22 & 23.29 & 26.03 \\
\hline Direcciones Generales & 13.75 & 11.49 & 14.15 & 16.75 & 16.36 & 15.79 & 23.35 & 18.33 & 26.48 \\
\hline
\end{tabular}

Fuente: Instituto de la Mujer

En tercer lugar, son las subsecretarias, tras los ministerios, los que mayor crecimiento han experimentado, en concreto entre 1995 y 2007 han pasado del 3,09 al 26,03, es decir, su participación ha aumentado en estos departamentos en 22,94 puntos. Aquí no existen diferencias entre los gobiernos populares y los socialistas, ya que la tendencia al alza se inicia con los primeros y continúa con los segundos, estableciéndose solamente una diferencia entre unos y otros de 3,81 puntos. Otra cuestión sería las funciones que se han dedicado a desempeñar en los mismos, principalmente básicas y socioculturales, es decir, en principio espacios más abiertos, que las económicas e infraestructuras que parecen estar más reservadas al público masculino.

Tabla 11. Altos cargos en la Administración Pública por funciones

\begin{tabular}{|l|c|c|c|c|}
\cline { 2 - 5 } \multicolumn{1}{c|}{} & $\mathbf{2 0 0 2}$ & $\mathbf{2 0 0 4}$ & $\mathbf{2 0 0 6}$ & $\mathbf{2 0 0 7}$ \\
\hline Ministros & $\mathbf{3 . 7 0}$ & $\mathbf{5 0}$ & $\mathbf{4 7 . 0 6}$ & $\mathbf{4 3 . 7 5}$ \\
\hline Funciones Básicas & 20 & 16.67 & 14.29 & 33.33 \\
\hline Funciones Económicas & & 33.33 & 33.33 & 33.33 \\
\hline Infraestructura & & 100 & 100 & 100 \\
\hline Funciones Socioculturales & & 75 & 73 & 25 \\
\hline Secretaría & $\mathbf{2 7}$ & $\mathbf{1 2}$ & $\mathbf{2 2 . 5 8}$ & $\mathbf{2 0}$ \\
\hline Funciones Básicas & 14 & 7.14 & 21.43 & 20 \\
\hline Funciones Económicas & 5 & & & \\
\hline Infraestructura & 2 & & 22.22 & \\
\hline Funciones Socioculturales & 6 & 40 & 50 & 40 \\
\hline Subsecretarias & $\mathbf{2 2 . 0 6}$ & $\mathbf{2 2 . 2 2}$ & $\mathbf{2 4 . 5 8}$ & $\mathbf{2 5}$ \\
\hline Funciones Básicas & 16.28 & 15.38 & 14.63 & 17.50 \\
\hline Funciones Económicas & 33.33 & 26.67 & 28.57 & 27.78 \\
\hline Infraestructura & 50 & 37.5 & 50 & 38 \\
\hline Funciones Socioculturales & 22.22 & 30 & 30 & 40 \\
\hline
\end{tabular}

Fuente: Instituto de la Mujer

En cuarto y último lugar estarían las secretarías de Estado, que suponen el ámbito donde las mujeres siguen teniendo mayores dificultades para incorporarse a la vida política, consiguiendo su mejor porcentaje durante la última legislatura de Felipe González con el $25 \%$. Es más, durante los gobiernos del PP, los porcentajes de mujeres en este espacio político no llegaron a superar en ninguno de los casos el 5\%. Pero prácticamente, lo mismo sucede durante los gobiernos socialistas, es cierto que las mujeres logran unos mayores porcentajes, pero suelen desempeñar funciones básicas, lo que nos indica dos cosas: la política de cuotas y la Ley de Igualdad no están siendo completamente 
respetadas, y 2) los sistemas de cuotas en estos casos no inciden realmente en una verdadera integración de las mujeres en estos organismos, sino que más bien supone un aumento en el número de las mismas pero sin concederles grandes funciones ni poderes. No obstante, son los gobiernos socialistas los que han jugado un papel decisivo en el incremento de mujeres en las Secretarias de Estado como demuestra el hecho de que entre 2004 y 2007 la presencia femenina en este tipo de cargos haya aumentado en casi nueve puntos.

\section{c) El papel de las mujeres en el Parlamento Europeo}

También en el Parlamento Europeo podemos comprobar el efecto que ha producido en las listas electorales los sistemas de cuotas en 1989 y 1997, y en este caso, incluso el de la Ley de Igualdad. Así, las mujeres socialistas han pasado de tener una representación del $11 \%$ en 1987 al 19\% en 1989, mientras que en 2004 la misma llegó a situarse en el 44\%. No obstante, también hay que reconocer que la participación de las mujeres ha sido tradicionalmente más alta en el Parlamento Europeo que en el Congreso, y mayor en el Congreso que en el Senado. Lo que significa que cuanto más proporcional sea el sistema electoral y cuantos más representantes se elijan en las circunscripciones, más mujeres obtienen cargos públicos. Pero también cuanto mayor poder tiene un organismo menor es la presencia de las mujeres en el mismo.

Tabla 12. Porcentaje y número de mujeres en el Parlamento Europeo, 1987/2008

\begin{tabular}{|l|c|c|c|c|c|c|}
\cline { 2 - 7 } \multicolumn{1}{c|}{} & $\mathbf{1 9 8 7}$ & $\mathbf{1 9 8 9}$ & $\mathbf{1 9 9 4}$ & $\mathbf{1 9 9 9}$ & $\mathbf{2 0 0 4}$ & $\mathbf{2 0 0 8}$ \\
\hline PSOE & $11(3)$ & $19(5)$ & $36(8)$ & $42(10)$ & $44(11)$ & $42,8(9)$ \\
\hline PP & $6(1)$ & $7(1)$ & $29(8)$ & $37(10)$ & $29(7)$ & $34,7(8)$ \\
\hline
\end{tabular}

Fuente: Elaboración propia

Por otra parte, según los datos que nos aporta la tabla 12, podemos comprobar como de nuevo las mujeres socialistas están más representadas que las populares en la Eurocámara, sólo en una ocasión, en 1999, parece que hubo un cierto equilibro entre ambas fuerzas políticas. El motivo es que la victoria electoral nacional del PP en 1996, supuso la necesidad de tener que cubrir de muchos cargos institucionales (secretarias de Estado, direcciones generales, etc.), y esto indudablemente ocasionó que las listas al Parlamento Europeo estuvieran menos codiciadas que en otros casos. Por tanto, era más fácil poder incorporar a las mujeres en la candidatura del partido para estos comicios. Como demuestra el hecho que tras la derrota electoral en las elecciones generales de 2004, el número de mujeres populares en la eurocámara volviera a descender. No obstante, también hay que recalcar otro dato, la Ley de Igualdad no ha conseguido los efectos esperados, teniendo en cuenta que en ninguno de los dos casos las mujeres han alcanzado el 50\% de la representación, es más en las elecciones europeas de 2009 la presencia de mujeres en el grupo socialista descendió en dos puntos.

\section{CONCLUSIONES}

El incremento de la representación femenina se ha producido tanto por cuestiones electorales como presiones internas. Primero, porque indudablemente los partidos políticos 
con aspiraciones de llegar al gobierno no pueden desoír las demandas de un sector importante de la población que tiene derecho al voto. Segundo, porque las mujeres que son militantes de los partidos políticos, forman parte de esta sociedad, y también empiezan a exigir nuevos canales de participación. Aunque igualmente habría que reconocer que la ideología de los partidos ha sido determinante en este proceso. Efectivamente, los partidos de izquierda han mostrado una mayor predisposición para establecer y poner en marcha estrategias que vinieran a favorecer las políticas de discriminación positiva. Pero habría que recalcar que éstas no serán del todo efectivas sino se llevan a cabo medidas que tiendan a proporcionar a las mujeres la disponibilidad necesaria para poder desarrollar esta actividad, o mejor dicho, para que puedan ser capaces de compatibilizar su profesión con su obligación familiar. Cosa, por otra parte, que no resulta sencilla, si en los partidos, como en cualquier ámbito profesional, no se empieza a ofrecer la flexibilidad necesaria para que puedan compaginar ambas funciones. De ahí, que sea tan importante establecer el sistema de cuotas como introducir medidas de conciliación familiar. Por eso, los socialistas afirman que la actividad del partido no puede realizarse sin tener en cuenta las dificultades que en la sociedad actual tienen las militantes para participar plenamente, horarios familiares, carencia de guarderías, etc. Por tanto, el partido tiene en cuenta estas situaciones, y apuesta por lograr la igualdad de oportunidades y la participación efectiva de las mujeres. En el PP prácticamente sucede lo mismo, el partido apuesta por ir introduciendo medidas que permitan conciliar ambos mundos, no obstante, el gobierno popular fue el primero en crear guarderías en los centros e instituciones públicas, como los ministerios. El problema es que luego la realidad es otra muy distinta, y las mujeres, a parte de enfrentarse a las barreras propias de su actividad profesional, tienen que afrontar otras dificultades añadidas como la conciliación familiar, que en muchos de los casos son un verdadero lastre para asumir unos puestos de mayor responsabilidad que conllevan una mayor dedicación temporal.

En todo caso, ni el sistema de cuotas ni la Ley de Igualdad, a la vista de los resultados obtenidos, parecen haber cumplido completamente con sus cometidos, dado que a día de hoy las mujeres aún están lejos de lograr una representación paritaria real en las esferas del poder. Además, otro hecho a tener en cuenta es que pese a la incorporación de las mujeres a puestos de poder, éstas presentan grandes diferencias salariales y de funciones con sus homólogos hombres, salvo claro está en la Administración Pública. Porque se puede cumplir con la norma sin ajustarse al espíritu de la misma, ya que se coloca a las mujeres al final de las listas, o bien a lo largo de todas ellas, unos de los principales retos que todavía tenemos, aunque estamos avanzando, es superar las desigualdades históricas entre hombres y mujeres. Y para ello, será necesario que se establezcan todo tipo de políticas de discriminación positiva, porque al fin y al cabo las cuotas de participación femenina constituyen un mecanismo que fuerza la integración de las mujeres en la vida política. Pero evidentemente la efectividad de su aplicación depende en buena medida de otros elementos, tales como la disposición de los dirigentes partidarios para abrir los espacios de decisión y candidaturas al género femenino. De esta manera, las cuotas sólo prosperaran y alcanzaran su mayor impacto cuando las mujeres hayan logrado penetrar en las estructuras de los partidos y colocarse en los niveles de mando intermedio y superior, aunque el tipo de sistema electoral también afectará al buen funcionamiento de las cuotas. En este sentido, cabría decir que por lo general, las cuotas tienden a funcionar mejor en sistemas de representación proporcional que en los de mayoría donde solamente hay un candidato por 
circunscripción. Esto no significa que sin esta estrategia no se pueda seguir avanzando hacia la paridad y la igualdad, pero si que se hará a un menor ritmo. Con esto tampoco estamos queriendo decir, que haya que integrar a mujeres en puestos de máxima relevancia por el hecho de ser mujer, sino que hay que permitir a éstas que puedan acceder a ellos siempre y cuando acrediten los méritos necesarios para desempeñar las funciones que les van a ser encomendadas. Lo ideal sería que las mujeres pudieran competir en igualdad de condiciones y respetándose unas mismas reglas de juego.

Otra conclusión a la que hemos podido llegar es que todos los partidos políticos, en mayor o menor medida, están integrando paulatinamente a las mujeres tanto en sus órganos de dirección como en los niveles institucionales donde logran representación. Unos, apuestan por el sistema de cuotas, como en el PSOE, mientras que otros, como el $\mathrm{PP}$, simplemente se limitan a defender la valía y los méritos para integrar a las mujeres. Aunque, según el estudio realizado, podemos afirmar que han sido los partidos que han regulado estatutariamente medidas de discriminación positiva los que han obtenido mejores resultados a este respecto, ya que, como hemos podido comprobar, en las fuerzas políticas que no han establecido ningún sistema de cuotas, como en el caso del PP, las mujeres han visto incrementada su presencia en la organización y en sus ámbitos de representación pero en menor medida que lo han hecho los que si han introducido este tipo de técnicas. En cualquier caso, nos estamos refiriendo a un impacto que tiene un carácter más cuantitativo que cualitativo, porque realmente, salvo en el último gobierno de José Luis Rodríguez Zapatero, las mujeres suelen ser relegadas a cometidos de segundo orden y alejadas de aquellas competencias predominantemente masculinas. Como demuestra el hecho, de que rara vez las mujeres ocupen las carteras ministeriales de defensa, economía e interior, o que sean designadas como secretarias generales o presidentas de los partidos, o que en la administración desempeñen funciones económicas o de infraestructuras. Aunque en cualquier caso, y pese a todas las deficiencias que todavía podamos encontrar, los sistemas de cuotas o la Ley de Igualdad han venido a favorecer la incorporación de las mujeres a la vida pública, si bien todavía queda mucho camino que correr para lograr una paridad real entre hombres y mujeres.

\section{BIBLIOGRAFÍA}

BAÓN, R. (1999): Historia del PP. Del franquismo a la Refundación, Madrid, Safel de Ibersaf Editores.

BATAILlE, P. y GASPARD, F. (2000): Cómo las mujeres cambian la política y por qué los hombres se resisten, Buenos Aires, Ediciones de la Flor.

BUTER, A. E. (1999): Talking feminist politics. New York. Rowman \& Littlefield Publishers.

CERNUDA, P. (2000): La mujer en la política. El largo camino hacia la igualdad, Barcelona, Plaza y Janés.

DELPHY, C. (1991) : "Penser le genre: quels problémes?”, en Hurting, M.C., Kail, M. y Rouch, H. (eds.). Sexe et genre. De la hiérarchie entre les sexes, Paris, CNRS, pp. 89-101.

GARCÍA DE LEÓN, M. A. (2001): Las mujeres políticas españolas (un ensayo sociológico), Madrid, Dirección General de la Mujer, Consejería de Presidencia de la Comunidad de Madrid.

HALL, R. (1973): Organizaciones: Estructura y procesos, Nueva Jersey. Editorial Prentice-Hall International.

HARMEL, R y JANDA, K. (1994): “An integrated theory of party goals and a party change", Journal of Theoretical Politics, $\mathrm{n}^{\circ} 6$ (3), pp. 259/287. 
KATZ, R. S. y MAIR, P. (1994): How parties organize. Change and adaptation in party organizations in western democracies, London, Sage Publications.

PANEBIANCO, A. (1990): Modelos de partido, Madrid, Alianza Universidad.

RUIZ JIMÉNEZ, Ma . A. (2002): Mecanismos del cambio ideológico e introducción de políticas de género en partidos conservadores: el caso de AP/PP en España en perspectiva comparada, Madrid, Instituto Juan March de Estudios de Investigaciones.

SÁNCHEZ, I. (2000): "PP y PSOE renueva más de la mitad de sus candidatos. Mujeres y novatos en los escaños del 2000", El Siglo de Europa, n 14, pp. 22/23.

SÁNCHEZ MEDERO, G. (2008): "El papel de las mujeres en la políticas española: el caso del PSOE", Revista de Estudios Feministas, vol. 16, n 2, octubre, pp. 433/462.

URIARTE, E. (1994) "Las transformaciones en el papel de la mujer", Inguruak, Revista de Sociología, $\mathrm{n}^{\mathrm{o}} 10$, noviembre, pp. 221/237.

- (1995): “Mujer y política en España”, Sistema, no 124, pp. 121/136.

URIARTE, E. y ELIZONDO, A. (coord.) (1997): Mujeres de política, Barcelona, Ariel.

VERGE, T. (2005): Mujer y partidos políticos en España: las estrategias de los partidos y su impacto institucional, 1978/2004", en VII Congreso Español de Ciencia Política y de la Administración, "Democracia y Buen Gobierno", en Madrid, entre 21/23 de septiembre.

- (2005): Partidos y representación política: las dimensiones del cambio en los partidos políticos españoles, 1977-2004. Madrid, Instituto Universitario de Investigación Ortega y Gasset-Universidad Complutense. Tesis Doctoral.

- (2006): "Mujer y partidos políticos en España: las estrategias de los partidos políticos y su impacto institucional, 1978-2004", REIS, n 115, pp. 165/196.

VERSTRYNGE, J. (1999): Memorias de un maldito, Barcelona, Grijalbo Mondadori-Hojas Nuevas.

\section{Breve currículo:}

Doctora en Ciencias Políticas por la Universidad Complutense de Madrid. Ha publicado distintos artículos: "Frente al Imperio (Guerra Asimétrica y Guerra Total"; "Política de Inmigración en España (1985-2000): Una Perspectiva Intergubernamental"; "Evaluación de la Acción y de las Políticas Públicas. Una visión global desde la bibliografia", "PP \& CDS. Pactos y Alternativas de Gobierno de Centro-Derecha en 1989", "Inmigración, capitalismo, proteccionismo e identidad II: el caso español”, "Resistencia y guerra asimétrica”, "Los partidos políticos españoles”, “¿Qué sucedió el 2 de diciembre de 2007 en Venezuela?”, "¿Quién ganó las elecciones autonómicas y municipales celebradas el 27 de mayo de 2007 en España?", "El centro mató a la izquierda", "Una respuesta serena a los detractores del Informe sobre Inmigración en España", " $21^{\text {st }}$ Century to two new challeges: Cyberwar and Cyberterrorism", "El PP ante su XVI Congreso Nacional: se rompe la tónica imperante", etc, además participar en diversos proyectos de investigación algunos de ellos dentro del programa $\mathrm{i}+\mathrm{d}$ regionales y nacionales. 